\title{
Genetic recombination of bovine viral diarrhea virus subgenotype -1a and $-1 c$ in persistently infected dairy cattle
}

\author{
Sri Handayani Irianingsih ${ }^{1,{ }^{*}}$, Bagoes Poermadjaja $^{1}$, Hastari Wuryastuti $^{2}$, Raden Wasito $^{2}$ \\ ${ }^{1}$ Disease Investigation Centre Wates, Jl. Raya Yogya-Wates Km. 27, Tromol Pos 18, Wates, Yogyakarta 55602, Indonesia \\ ${ }^{2}$ Faculty of Veterinary Medicine, Universitas Gadjah Mada, Jl. Fauna No.2, Sleman, Yogyakarta 55281, Indonesia \\ *Corresponding author: shirianingsih@gmail.com
}

SUBMITTED 7 February 2020 REVISED 20 May 2020 ACCEPTED 20 September 2020

\begin{abstract}
The bovine viral diarrhea virus (BVDV) is a major viral pathogen in cattle worldwide. In Indonesia, diversity in subgenotypes of BVDV-1 has been observed, with the highest proportion of subgenotype -1a, followed by $-1 \mathrm{c},-1 \mathrm{~b}$, and $-1 \mathrm{~d}$. So far, phylogenetic analysis of BVDV-1 is based on nucleotide sequences of the $5^{\prime}$ UTR and partial NS5B regions. Accuracy in identifying the subgenotype and antigenic type is critical for vaccine development and effective vaccination. The aim of this study was to determine genetic recombination of BVDV through phylogenetic analysis of five different regions (5' UTR, NPro, E2, NS3, and NS5B) of BVDV in persistently infected dairy cattle. Five isolates were sequenced using next-generation sequencing, and data were analyzed with the CLC Genomic Workbench 9.0 and MEGA-X programs. Phylogenetic analysis based on the 5' UTR (275 nt), NPro (504 nt), E2 (1,122 nt), NS3 (2,049 nt), and NS5B (2,157 nt) regions indicated that one BVDV isolate from Banyumas, Central Java, could be classified into different subgenotypes based on the E2 region (-1c), but the same subgenotype based on the other four regions (-1a), suggesting the presence of genetic recombination of the BVDV subgenotypes - $1 \mathrm{a}$ and $-1 \mathrm{c}$ in persistently infected dairy cattle.
\end{abstract}

KEYWORDS BVD virus; persistent infection; phylogenetic; recombination; subgenotype

\section{Introduction}

Bovine viral diarrhea virus (BVDV) is an important viral pathogen in cattle that has spread throughout the world and has an economic impact with respect to animal husbandry (Houe et al. 1995; Deregt et al. 2005). BVDV was first reported in 1946 in North America and has now expanded throughout the Americas and into Europe, Australia, Africa, and Asia, including Indonesia (Wiyono et al. 1989; Brodersen 2014). The incidence of BVDV in Indonesia was first described in Balinese cattle in South Sulawesi in 1989 (Wiyono et al. 1989).

Persistent infection (PI) in cattle occurs when the noncytopathic BVDV infects pregnant cattle during the first trimester (40-120 d) of gestation, when the fetal immune system has not completely developed (Neill 2013). PI in cattle is determined by collecting blood samples twice at intervals of at least 3-4 weeks, after which PI is detected using antigens (Firat et al. 2002). Persistently infected cattle are a potential source of transmitting the virus and therefore, the disease, to herd communities (LiebnerTenorio 2005).

BVDV is a member of the genus Pestivirus, and the family Flaviviridae. It is a small, approximately 12.3 kb-long, enveloped, single-stranded positive-sense RNA virus (Chernick and van der Meer 2017). The genome of BVDV contains one open-reading frame that encodes a large polyprotein with the sequence NPro-C-E1-E2-p7NS2-NS3-NS4a-NS4b-NS5a-NS5b (Neill 2013). BVDV has two genotypes: BVDV-1 and BVDV-2 (Nagai et al. 2004; Vilcek et al. 2005). Genotype classification of BVDV is usually based on comparison of sequences from three genetic regions: 5' UTR, Npro, and E2. Initially, there were only two subgenotypes of BVDV-1 isolates identified, namely, BVDV-1a and BVDV-1b (Vilcek et al. 2005). However, based on different regions (5' UTR, Npro, E2), BVDV-1 is now divided into 21 subgenotypes (BVDV-1a 1u) (Gao et al. 2014; Yeşilbağ et al. 2014; Bazzucchi et al. 2017); whereas BVDV-2 has four subgenotypes, probably owing to the lack of analyzed virus collections (Vilcek et al. 2005; Giangaspero et al. 2008).

The distribution of BVDV-1 in Indonesia is indicated by a number of samples from Java that have been evaluated based on three genes: NS5B, NPro, and 5' UTR (Saepulloh et al. 2015; Karimy 2016; Wuryastuti et al. 2018). Meanwhile, BVDV-2 has not been found in individual samples of BVDV-PI from Central Java. It is believed that there are two main BVDV-1 subgenotypes, -1a and -1c (Saepulloh et al. 2015; Wuryastuti et al. 2015, 2018), in addition to 
other subgenotypes, $-1 \mathrm{~b}$ and -1d (Saepulloh et al. 2015). The distribution of three subgenotypes, namely, BVDV$1 \mathrm{a},-1 \mathrm{~b}$, and $-1 \mathrm{c}$, in Central and East Java, Indonesia, reflects the genetic variability of BVDV-1 viruses based on partial NS5B genes (Irianingsih et al. 2019). Phylogenetic analysis from different regions, specifically 5' NCR, Npro, E2, NS3, and NS5B-3' NCR, of BVDV isolates has been carried out in Japan (Nagai et al. 2004) and 5'UTR nd NPro in the UK (Booth et al. 2013). The determination of BVDV genotypes and subgenotypes is very useful for the correct classification of BVDV for the purposes of diagnosis, identification, and characterization of viruses, as well as for evaluating vaccine efficacy and molecular epidemiology (Nagai et al. 2004; Vilcek et al. 2005). This study seeks to determine genetic recombination in BVDV through phylogenetic analysis based on five different regions, namely, non-coding regions (NCR): 5' UTR, nonstructural genes (NPro, NS3, and NS5B), and structural genes (E2) of BVDVs from persistently infected dairy cattle.

\section{Materials and Methods}

\subsection{Virus isolation}

Five BVDV isolates were obtained from serum samples of persistently infected dairy cattle in Central and East Java. These were collected by active and passive surveillance by the Disease Investigation Centre Wates, over the course of 2013 2016. Serum samples were inoculated in MDBK cells that were incubated at $37^{\circ} \mathrm{C}$ in $5 \% \mathrm{CO}_{2}$ in a maintenance medium (Minimal Essential Medium, MEM, supplemented with $2 \%$ bovine serum, penicillin-streptomycin $100 \mathrm{IU} / \mathrm{mL}-100 \mu \mathrm{g} / \mathrm{mL}$, gentamycin $50 \mu \mathrm{g} / \mathrm{mL}$, fungizone $2.5 \mu \mathrm{g} / \mathrm{mL}$, and HEPES buffer $0.01 \mathrm{M}$ ) for 3-5 d. Virus isolation required four passages in cell culture and then identification using real-time RT-PCR on BVDV. Virus isolates were stored in a freezer $\left(-80^{\circ} \mathrm{C}\right)$ until subsequent experiments.

\subsection{Targeted sequencing}

RNA from BVDV isolates was extracted using the QIAamp Viral RNA kit (Qiagen, Germany). Using RNA as a template, single-stranded cDNAs were generated with the SuperScript III First-Strand Synthesis System for RTPCR (Invitrogen, California, USA) according to the man- ufacturer's instructions. The BVDV genome was amplified using a series of four primers that overlapped fragments of the protein-coding region (Chernick and van der Meer 2017) employing Platinum Taq DNA Polymerase High Fidelity (Invitrogen, California, USA). The amplicons were gel-purified using the DNA Clean \& Concentrator (Zymo Research, California, USA) and quantified with the Qubit 2.0 and Qubit ds DNA HS Assay kits (Thermo Fisher Scientific, Waltham, US). Amplicons were pooled into equimolar amounts and adjusted for amplicon length. Each pool was subjected to library preparation using a Nextera XT DNA Library Preparation kit (Illumina, San Diego, USA). All libraries were prepared with dual indices and sequenced with paired-end, 300-bp reads on a MiSeq using a 600-cycle V3 cartridge (Illumina, San Diego, USA). The NGS approach has advantages, by producing massive sequencing data, decreasing costs, and performing high throughput analysis compare to conventional sequencing. Sequencing was conducted in the biotechnology laboratory of the Disease Investigation Center Wates, Yogyakarta, Indonesia.

\subsection{Phylogenetic analysis}

Raw reads were imported into the CLC Genomics Workbench (Qiagen) for pre-processing and assembly. Reads were paired and assembled with CLC Genomics set to map reads to reference. The accession number, KF896608, was used as a reference for mapping. Consensus sequences were extracted from the assemblies for all single sequences. Gene-specific consensus sequence alignments of five different regions (5' UTR, NPro, E2, NS3, and NS5B) were constructed using MUSCLE in MEGA$\mathrm{X}$. Phylogenetic analysis of the five regions was performed utilizing the Maximum-Likelihood method and bootstrap test $(n=1,000)$. The BVDV sequences available in GenBank were aligned with the sample sequence using blast-n for phylogenetic analysis.

\section{Results and Discussion}

\subsection{Result}

Five BVDV isolates were sequenced using the NGS technique with MiSeq Illumina. The sequencing results were then assessed for five different regions, namely 5' UTR, NPro, E2, NS3, and NS5B to determine the BVDV

TABLE 1 Summary on the sequencing results of five sample on five regions of the BVDV with next generation sequencing techniques.

\begin{tabular}{|c|c|c|c|c|c|}
\hline Sample Code & $\begin{array}{l}\text { 5'UTR } \\
\text { (275 nt) }\end{array}$ & $\begin{array}{l}\text { NPro } \\
\text { (504 nt) }\end{array}$ & $\begin{array}{l}\text { E2 } \\
(1,122 n t)\end{array}$ & $\begin{array}{l}\text { NS3 } \\
(2,049 \mathrm{nt})\end{array}$ & $\begin{array}{l}\text { NS5B } \\
(2,157 \mathrm{nt})\end{array}$ \\
\hline V1 BVDV1/Indonesia-EJ-Psn/04131368-2/2013 & full & full & full & full & partial $(1,038 \mathrm{nt})$ \\
\hline V4 BVDV1/Indonesia-CJ-Bms/HST-0783/2014 & full & full & partial (385 nt)* & none* & full \\
\hline V7 BVDV1/Indonesia-CJ-Bms/04151167-16/2015 & full & full & full & full & full \\
\hline V8 BVDV1/Indonesia-CJ-Bms/04151167-17/2015 & full & full & full & partial (391 nt)* & partial (244 nt)* \\
\hline V20BVDV1/Indonesia-CJ-Bms/04161644-14/2016 & full & full & partial (1,039 nt) & none* & partial $(1,965 \mathrm{nt})$ \\
\hline
\end{tabular}

*phylogenetic analysis was not followed 


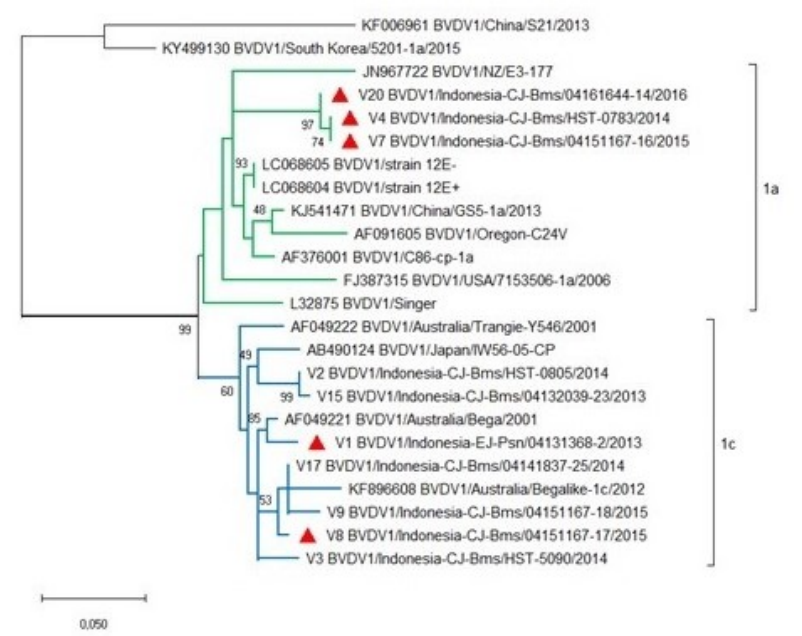

FIGURE 1 A phylogenetic tree based on the 5'UTR (275 nt) region with Maximum Likelihood method, Kimura-2 parameter model, and $1,000 x$ bootstrap number. Samples were marked with red triangle.

subgenotype. Details of the sequencing results for the five samples are shown in Table 1.

Based on the sequencing results, the full length of the 5' UTR and NPro genes could be analyzed, whereas there were two to four samples for genes E2, NS3, and NS5B. Sample sequences with only $30-45 \%$ of whole sequences were less representative of the region. Moreover, these five regions are categorized into untranslated regions (5' UTR), structural genes (E2), and nonstructural genes (NPro, NS3, and NS5B). The phylogenetic tree of BVDV-1 based on the 5' UTR along 275 nucleotides (nt) against five BVDV isolates are shown in Figure 1. BVDV-1 was divided into two subgenotypes-1a and -1c, with 99\% bootstrap values. Three Indonesian isolates, V4 BVDV1/Indonesia-CJ-Bms/HST-0783/2014, V7 BVDV1/Indonesia-CJ-Bms/04151167-16/2015, and V20 BVDV1/Indonesia-CJ-Bms/04161644-14/2016 had same branch with $97 \%$ bootstrap values. They were clustered with sequences L32875 BVDV1/Singer and AF091605 BVDV1/Oregon-C24V as BVDV-1a references obtained from Genbank. Two other isolates, V1 BVDV1/Indonesia-EJ-Psn/04131368-2/2013 and V8 BVDV1/Indonesia-CJ-Bms/04151167-17/2015 were included in subgenotype-1c with KF896608 BVDV1/Australia/Begalike-1c/2012 and AF049221 BVDV1/Australia/Bega/2001.

The nucleotide sequences of five field viruses and a number of known reference strains originating from GenBank were aligned and phylogenetic trees were constructed. Phylogenetic trees based on non-structural regions of NPro along $504 \mathrm{nt}$ against five BVDV isolates were shown in Figure 2. Two subgenotypes-1a and -1c were also divided the same as phylogenetic tree of 5'UTR. The BVDV-1a branch was supported by $97 \%$ bootstrap value. It showed three isolates V4 BVDV1/IndonesiaCJ-Bms/HST-0783/2014， V7 BVDV1/Indonesia-CJBms/04151167-16/2015, and V20 BVDV1/Indonesia-

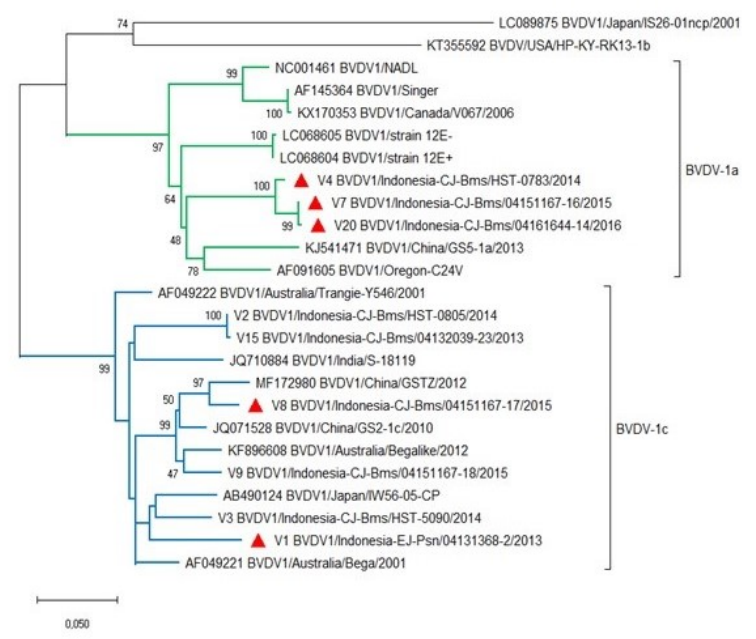

FIGURE 2 A phylogenetic tree based on the full length of NPro region (504 nt) with the Maximum Likelihood method, Tamura3 parameter model, and 1,000x bootstrap numbers. Samples are marked with red triangle.

CJ-Bms/04161644-14/2016 in one group with AF091605/BVDV-1/Oregon-C24V, NC001461 BVDV1/NADL, and AF145364 BVDV1/Singer as BVDV1-a references. Two other Indonesian isolates, V1 BVDV1/Indonesia-EJ-Psn/04131368-2/2013 and V8 BVDV1/Indonesia-CJ-Bms/04151167-17/2015 were in a separate branch supported by $99 \%$ bootstrap value as subgenotype - 1 c. They were included in with KF896608 BVDV1/Australia/Begalike-1c/2012, AF049221 BVDV1/Australia/Bega/2001 and AF049222 BVDV1/Australia/Trangie-Y546/2001.

The sequences of 2,049 nt of nonstructural region of NS3 were determined and analyzed with 18 sequences data from GenBank (Figure 3). Two Indonesian BVDV1 isolates could be analyzed in full length. There were two branch in this phylogenetic tree constructed the same branch as that of 5'UTR and NPro regions, BVDV-1a and BVDV-1c with a bootstrap value of $97 \%$. One isolate, V7 BVDV1/Indonesia-CJ-Bms/0415116716/2015 was clustered as subgnotype BVDV-1a with NC001461/BVDV-1/NADL and other BVDV1-a references. Isolate V1 BVDV1/Indonesia-EJ-Psn/041313682/2013 from different district clustered phylogenetically with BVDV-1c references, KF896608/Australia/Begalike/2012 and AF052304 Pest type 1 strain Trangie. The sequence of three isolates could not be analyzed in NS3 region because the length of sequence had no good results, either no sequence or less than $50 \%$.

The phylogenetic tree based on the non-structural regions of NS5B along 2,157 nt against four BVDV isolates and 21 sequences originating from Genbank is shown in Figure 4. The phylogenetic tree of this region also showed the same topology as the 5'UTR, NPro, and NS3. There were four Indonesian BVDV-1 isolates which divided into two subgenotypes-1a and -1c with a bootstrap value of $97 \%$. Subgenotype-1a showed three isolates V4 BVDV1/Indonesia-CJ-Bms/HST-0783/2014, 


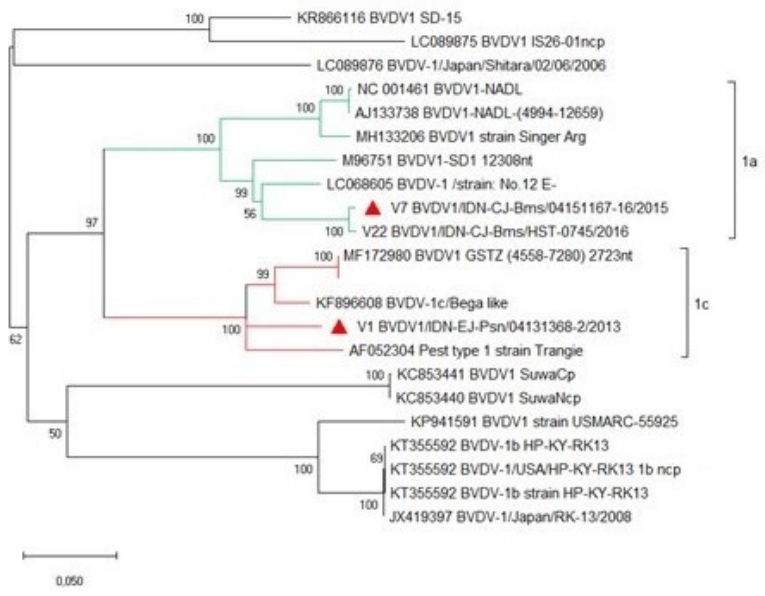

FIGURE 3 A phylogenetic tree based on the NS3 region (2,049 nt) with the Maximum Likelihood method, the General Time Reversible model, and a 1,000x bootstrap number. Samples are marked with red triangle.

V7 BVDV1/Indonesia-CJ-Bms/04151167-16/2015, and V20 BVDV1/Indonesia-CJ-Bms/04161644-14/2016 were in one group with AF091605/BVDV-1/OregonC24V, NC001461 BVDV1/NADL, and AF145364 BVDV1/Singer. One isolate, V1 BVDV1/Indonesia-EJPsn/04131368-2/2013 was included in subgenotype-1c with KF896608/Australia/Bega-like/2012. There was one isolate that could not be analyzed on this region because of less than $50 \%$ full length sequence.

The structural E2 region was the fifth region to be analysed of the following 1,122 bases. There were four Indonesia BVDV-1 isolates could be analysed, however one isolate was less than $50 \%$ of full length sequence. The phylogenetic tree based on the structural region of E2 along 1,122 nt against Indonesian BVDV-1 isolates and 20 sequences originating from Genbank is shown in Figure 5. This phylogenetic was constructed using the same references that generated for the first four phylogenetic analysis. Two subgenotypes, BVDV-1a and BVDV-1c were obtained from phylogenetic tree of E2 region BVDV-1 with a bootstrap value of $99 \%$. Three out of four isolates, V1 BVDV1/IndonesiaEJ-Psn/04131368-2/2013， V8 BVDV1/Indonesia-CJBms/04151167-17/2015, and V20 BVDV1/Indonesia-CJBms/04161644-14/2016 were clustered as subgenotype1c with KF896608 BVDV1/Australia/Begalike-1c/2012, AF049221 BVDV1/Australia/Bega /2001 and AF049222 BVDV1/Australia/Trangie-Y546/2001. One isolate, V7 BVDV1/Indonesia-CJ-Bms/04151167-16/2015 was included in the same subgenotype with AF091605/BVDV1/Oregon-C24V and NC001461 BVDV1/NADL as BVDV-1a references. There was one isolate V20 BVDV1/Indonesia-CJ-Bms/04161644-14/2016 was featured different subgenotypes, mostly -1c in the 5'UTR, NPro, NS3, and NS5B, while their E2 region were clustered into -1 a with $96 \%$ bootstrap value.

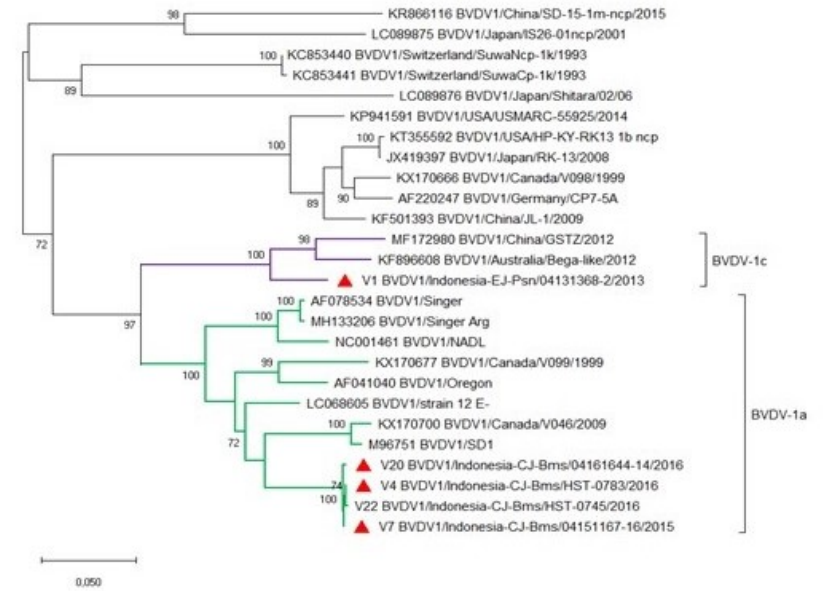

FIGURE 4 A phylogenetic tree based on the full length of NS5B region $(2,157 \mathrm{nt})$ with the Maximum Likelihood method, the General Time Reversible model and 1,000x bootstrap numbers. Samples are marked with red triangle.

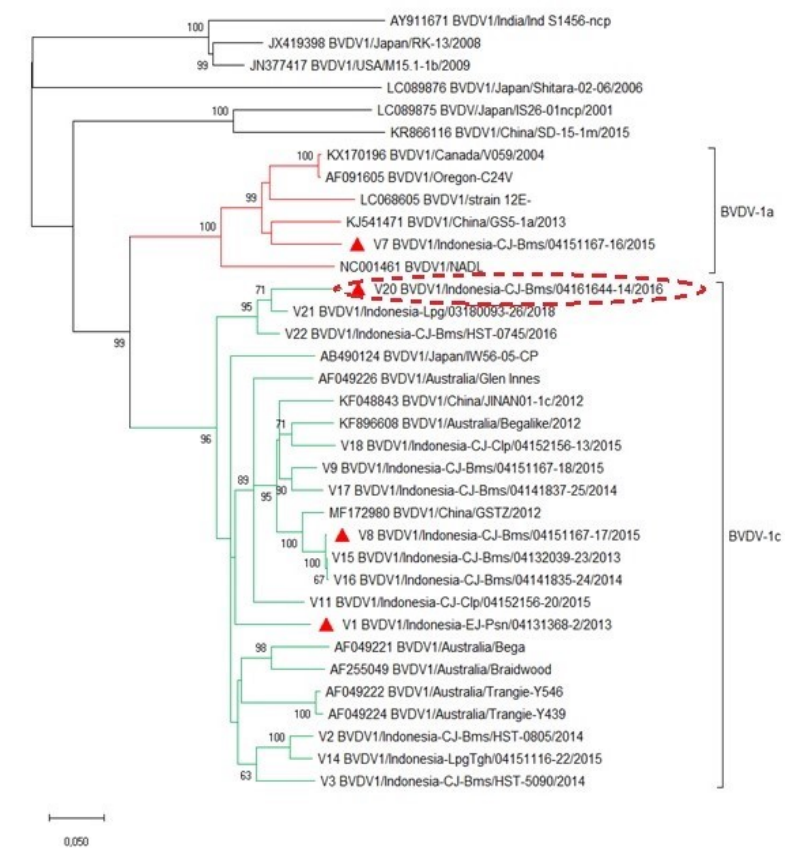

FIGURE 5 Phylogenetic tree based on the full length of E2 region (1,122 nt) with the Maximum Likelihood method, the General Time Reversible model, and 1,000x bootstrap. Samples are marked with red triangle. Sample with red dashed-line features a different subgenotype.

\subsection{Discussion}

Classification of subgenotypes of five BVDV isolates based on the five regions, 5 UTR, NPro, NS3, NS5B, and E2, resulted in two subgenotypes BVDV-1a and BVDV-1c, as per previously published studies in Indonesia (Wuryastuti et al. 2018). The 5'UTR phylogenetic tree using Australian BVDV reference sequences illustrated that three out of five Indonesian viruses belonged to the BVDV-1c. The other subgenotypes, $-1 \mathrm{~b}$ and $-1 \mathrm{~d}$ were not found in this study even though isolated from Central Java as previous studies (Saepulloh et al. 2015). 
According to Yeşilbağ et al. (2017), the comparison of global BVDV-1 distribution showed a greater distribution of BVDV-1b followed by BVDV-1a, whereas BVDV-1c was the most dominant subgenotype in Australia (Reichel et al. 2018). Comparison of BVD-1a subgenotypes based on 5' UTR is three-fold greater than BVD-1c in Java, Indonesia (Wuryastuti et al. 2018).

The conserved region could be targeted to detect various subgenotypes of BVDV (Nagai et al. 2004). Further genetic studies using American isolates (L32875 BVDV1/Singer, NC001461 BVDV1/NADL, and AF091605 BVDV1/Oregon-C24V) and Australian isolates (KF896608 BVDV1/Australia/Begalike-1c/2012, AF049221 BVDV1/Australia/Bega/2001 and AF049222 BVDV1/Australia/Trangie-Y546/2001) as references have shown that clustered BVDV-1a and BVDV-1c respectively. These findings are highly significant as published previously (Yeşilbağ et al. 2017) and also whole genome (Bazzucchi et al. 2017) that all references showed the appropriate subgenotype.

Isolates V4 BVDV1/Indonesia-CJ-Bms/HST0783/2014, V7 BVDV1/Indonesia-CJ-Bms/0415116716/2015, and V20 BVDV1/Indonesia-CJ-Bms/0416164414/2016 were clustered BVDV1-a based on the sequence data obtained from 5'UTR, NPro, NS3, and NS5B regions with 97-99\% bootstrap value. Based on the E2 region, one isolate V20 BVDV1/Indonesia-CJ-Bms/0416164414/2016 was clustered as BVDV1-c with two other isolates, V1 BVDV1/Indonesia-EJ-Psn/04131368-2/2013 and V8 BVDV1/Indonesia-CJ-Bms/04151167-17/2015 at a confidence level of $99 \%$ by bootstrap analysis. Isolate V20 BVDV1/Indonesia-CJ-Bms/04161644-14/2016 isolate had two subgenotypes BVDV-1a and BVDV-1c. The presence of antigenic variation of $\mathrm{E} 2$ protein among BVD virus strains contributes to vaccination failure (Ridpath 2005). The structural region, E2 as hypervariation region used to in this study for the phylogenetic analysis and could be used to predict the rate of mutations in the evolution and virulence of the virus as well as factors that influence the pathogenesis of the virus in causing disease (Goens 2002; Dow et al. 2015).

A collection of five BVDV isolates from persistently infected dairy cow samples was identified using real-time BVDV RT-PCR. Persistently infected cattle were the source of the virus, and thus have the potential to transmit BVDV; therefore, they excluded from the herd group (Liebner-Tenorio 2005). This persistent infection can be caused by infection in utero or infections that occur repeatedly because of environmental factors that are contaminated with viruses. Isolates V1 BVDV1/Indonesia-EJ-Psn/04131368-2/2013 and V8 BVDV1/Indonesia-CJ-Bms/04151167-17/2015 consistently belonged to subgenotype-1c with the reference sequence KF896608_Australia/Bega-like/2012. V4 BVDV1/Indonesia-CJ-Bms/HST-0783/2014, V7 BVDV1/Indonesia-CJ-Bms/04151167-16/2015, and V20 BVDV1/Indonesia-CJ-Bms/04161644-14/2016 isolates are in subgenotype -1a with LC068605/BVDV- 1a/strain 12E- and AF091605/BVDV-1a/Oregon-C24V based on the regions, 5' UTR, NPro, and NS5B, with high homology of nearly $98-100 \%$. One isolate, V20 BVDV1/Indonesia-CJ-Bms/04161644-14/2016, featured different subgenotypes, mostly $-1 \mathrm{c}$, based on the E2 region with $91 \%$ homology compared to other Indonesian virus isolates.

In this study, based on the time sequence, the 2013 virus isolate was identified as the BVDV-1c subgenotype, whereas in 2015, besides being identified as -1c, the isolate was also grouped in -1a. V20 BVDV1/Indonesia-CJBms/04161644-14/2016 isolates from 2016 cases showed the existence of both subgenotypes. According to Nagai et al. (2004), recombination between virus strains, classified into different subgroups, took place in animals through different subgenotypes. Genetic diversity can be caused by mutations, errors in RNA-dependent RNA polymerase activity, and recombination of homologous RNA and heterologous viral genomes; however, it is very important for taxonomy, laboratory diagnosis, and vaccine design (Vilcek et al. 2004).

Recombination in Pestiviruses has been characterized in BVDVs. The recombination can occur between the host and virus, producing a host RNA sequence that inserts into the viral genome or through rearrangement, duplication, or both, of the viral genome sequences (Goens 2002). Heterolog/non-homologous recombination in Pestiviruses is reported to produce cytopathic biotypes that can evolve from noncytopathic viruses and cellular sequences (Becher and Tautz 2011).

Homologous RNA recombination of the BVDV genome in structural regions has been observed in trials of animals infected with mucosal diseases (MDs) (Nagai et al. 2003). According to Peterhans and Schweizer (2010), persistently infected cattle are at risk of being infected with a mutant or different biotype BVDV, thus increasing their potential to have MD. The discovery of two subgenotypes, -1a and -1c, in V20 BVDV1/Indonesia-CJBms/04161644-14/2016 originating from persistently infected cattle indicates recombination of the viral genome and the potential to cause MD events. Phylogenetic analysis of various non-structural and structural protein-coding regions is useful for virus characterization and further epidemiological analysis.

\section{Conclusions}

Phylogenetic analysis of five BVDV isolates collected from persistently infected cattle was carried based on five different regions: the 5' UTR, nonstructural regions (NPro, NS3, NS5B), and the E2 structural region, and showed two subgenotypes, specifically $-1 \mathrm{a}$ and $-1 \mathrm{c}$. The analysis of one of these virus isolates also indicated genetic recombination of BVD subgenotypes -1a and -1c in persistently infected dairy cattle. 


\section{Acknowledgments}

The authors thank the Disease Investigation Centre Wates, the Directorate General of Livestock and Animal Health Services, Ministry of Agriculture, Republic of Indonesia for funding the development of methods and permission to use the BVDV isolate collection as research material.

\section{Authors' contributions}

This research was arranged, designed, and analyzed by SHI. BP, HW, and RW supervised the experiments and proofread the manuscript. All authors read and approved the final manuscript.

\section{Competing interests}

The authors declare no competing interest.

\section{References}

Bazzucchi M, Bertolotti L, Giammarioli M, Rossi E, Petrini S, Rosati S, De Mia GM. 2017. Complete genome sequence of a bovine viral diarrhea virus subgenotype $1 \mathrm{G}$ strain isolated in Italy. Genome Announc. 5(17):318-319. doi:10.1128/genomeA.00263-17.

Becher P, Tautz N. 2011. RNA recombination in pestiviruses: cellular RNA sequences in viral genomes highlight the role of host factors for viral persistence and lethal disease. RNA Biol. 8(2):216-224. doi:10.4161/rna.8.2.14514.

Booth RE, Thomas CJ, El-Attar LM, Gunn G, Brownlie J. 2013. A phylogenetic analysis of Bovine Viral Diarrhoea Virus (BVDV) isolates from six different regions of the UK and links to animal movement data. Vet Res. 44(1):43. doi:10.1186/1297-9716-44-43.

Brodersen B. 2014. Bovine viral diarrhea virus infections: manifestations of infection and recent advances in understanding pathogenesis and control. Vet Pathol. 51(2):453-464. doi:10.1177/0300985813520250.

Chernick A, van der Meer F. 2017. Evolution of Bovine viral diarrhea virus in Canada from 1997 to 2013. Virol. 509:232-238. doi:10.1016/j.virol.2017.06.024.

Deregt D, Dubovi EJ, Jolley ME, Nguyen P, Burton KM, Gilbert SA. 2005. Mapping of two antigenic domains on the NS3 protein of the pestivirus bovine viral diarrhea virus. Vet Microbiol. 108(1-2):13-22. doi:10.1016/j.vetmic.2005.02.010.

Dow N, Chernick A, Orsel K, van Marle G, van der Meer F. 2015. Genetic variability of bovine viral diarrhea virus and evidence for a possible genetic bottleneck during vertical transmission in persistently infected cattle. PloS one 10(7):e0131972. doi:10.1371/journal.pone.0131972.

Firat I, Ak S, Bozkurt HH, Ak K, Turan N, Bagcigil F. 2002. Distribution of bovine viral diarrhoea virus
(BVDV) in the genital system tissues of cattle. Vet Arhiv. 72(5):235-248.

Gao S, Du J, Shao J, Lang Y, Lin T, Cong G, Zhao F, Belák S, Liu L, Chang H, et al. 2014. Genome analysis reveals a novel genetically divergent subgenotype of bovine viral diarrhea virus in China. Infect Genet Evol. 21:489. doi:10.1016/j.meegid.2013.06.010.

Giangaspero M, Harasawa R, Weber L, Belloli A. 2008. Taxonomic and epidemiological aspects of the bovine viral diarrhoea virus 2 species through the observation of the secondary structures in the 5'genomic untranslated region. Vet Ital. 44(2):319-345.

Goens SD. 2002. The evolution of bovine viral diarrhea: a review. Can Vet J. 43(12):946.

Houe H, Baker J, Maes R, Wuryastuti H, Wasito R, Ruegg P, Lloyd J. 1995. Prevalence of cattle persistently infected with bovine viral diarrhea virus in 20 dairy herds in two counties in central Michigan and comparison of prevalence of antibody-positive cattle among herds with different infection and vaccination status. J Vet Diagn Invest. 7(3):321-326. doi:10.1177/104063879500700304.

Irianingsih S, Wuryastuty $\mathrm{H}$, Wasito $\mathrm{R}$, Wibawa $\mathrm{H}$, Rasa FT, Poermadjaja B. 2019. Genetic analysis of NS5B gene from bovine viral diarrhea virus-infected cattle in Central and East Java, Indonesia. Vet World. 12(7):1108. doi:10.14202/vetworld.2019.1108-115.

Karimy MF. 2016. Ragam genetik bovine viral diarrhea virus genotipe 1 (Singer dan CP) dan genotipe 2 (890, Ngawi, dan Ngambal) pada NPro Region. Master's thesis, Universitas Gadjah Mada, Yogyakarta.

Liebner-Tenorio EM. 2005. Pathogenesis. In: SM In: Goyal, e Ridpath Julia F, editors, Bovine viral diarrhea virus: diagnosis, management, and control. Iowa USA, John Wiley \& Sons, $1^{\text {st }}$ edition. p. 121143.

Nagai M, Hayashi M, Sugita S, Sakoda Y, Mori M, Murakami T, Ozawa T, Yamada N, Akashi H. 2004. Phylogenetic analysis of bovine viral diarrhea viruses using five different genetic regions. Virus Res. 99(2):103-113. doi:10.1016/j.virusres.2003.10.006.

Nagai M, Sakoda Y, Mori M, Hayashi M, Kida H, Akashi H. 2003. Insertion of cellular sequence and RNA recombination in the structural protein coding region of cytopathogenic bovine viral diarrhoea virus. J Gen Virol. 84(2):447-452. doi:10.1099/vir.0.18773-0.

Neill JD. 2013. Molecular biology of bovine viral diarrhea virus. Biol. 41(1):2-7. doi:10.1016/j.biologicals.2012.07.002.

Peterhans E, Schweizer M. 2010. Pestiviruses: how to outmaneuver your hosts. Vet Microbiol. 142(1-2):1825. doi:10.1016/j.vetmic.2009.09.038.

Reichel MP, Lanyon SR, Hill FI. 2018. Perspectives on Current Challenges and Opportunities for Bovine Viral Diarrhoea Virus Eradication in Australia and New Zealand. Pathogens 7(1):14. doi:10.3390/pathogens7010014.

Ridpath JF. 2005. Classification and Molecular Biology. 
In: SM In: Goyal, e Ridpath Julia F, editors, Bovine viral diarrhea virus: diagnosis, management, and control. Iowa USA, John Wiley \& Sons, $1^{\text {st }}$ edition. p. 65-80.

Saepulloh M, Sendow I, et al. 2015. Identification and characterization of bovine viral diarrhea virus from Indonesian cattle. J. Vet 16(1):1-7.

Vilcek S, Durkovic B, Kolesárová M, Greiser-Wilke I, Paton D. 2004. Genetic diversity of international bovine viral diarrhoea virus (BVDV) isolates: identification of a new BVDV-1 genetic group. Vet Res. 35(5):609615. doi:10.1051/vetres:2004036.

Vilcek S, Durkovic B, Kolesarova M, Paton D. 2005. Genetic diversity of BVDV: consequences for classification and molecular epidemiology. Prev Vet Med. 72(1-2):31-35. doi:10.1016/j.prevetmed.2005.08.004.

Wiyono A, Ronohardjo P, Graydon R, Daniels P. 1989. Diare ganas sapi: 1 . Kejadian penyakit pada sapi Bali bibit asal Sulawesi Selatan yang baru tiba di Kalimantan Barat. Penyakit Hewan 21(38):77-83.

Wuryastuti H, , Wasito R, Sugiyono. 2018. Genotypes and biotypes variation of bovine viral diarrhea virus from persistently infected dairy cattle in Java, Indonesia. Integr J Vet Biosci 3(2):1-7.

Wuryastuti H, Putro PP, Wasito R, Maes RK. 2015. Genetic Variability of Bovine Viral Diarrhea Virus in Persistently Infected Cattle in Central Java, Indonesia. Merit Res J Microbiol Biol Sci. 3(3):031-036.

Yeşilbağ K, Alpay G, Becher P. 2017. Variability and global distribution of subgenotypes of bovine viral diarrhea virus. Viruses 9(6):128. doi:10.3390/v9060128.

Yeşilbağ K, Förster C, Ozyiğit MO, Alpay G, Tuncer P, Thiel HJ, König M. 2014. Characterisation of bovine viral diarrhoea virus (BVDV) isolates from an outbreak with haemorrhagic enteritis and severe pneumonia. Vet Microbiol. 169(1-2):42-49. doi:10.1016/j.vetmic.2013.12.005. 